\author{
И. П. Лебедева \\ Российский экономический университет имени Г. В. Плеханова, \\ Москва, Россия
}

Вопросы, которые необходимо решить маркетологу при выпуске любого мобильного приложения, можно разделить на две группы. Первая группа связана с привлечением клиента, вторая - с его удержанием. С одной стороны, решение этих задач напрямую связано с проведением рекламной кампании и маркетинговых исследований, однако большая часть компаний - разработчиков мобильных приложений не имеет финансовых возможностей для маркетингового сопровождения выхода приложения на рынок. В данной статье рассмотрены исследования, которые можно проводить своими силами. При грамотном анализе они могут привести к качественному результату и увеличению количества пользователей. Кроме того, автором предложен метод анализа расстановки событий в программной части приложения, который помогает отслеживать действия пользователей и интерпретировать их для выявления основных проблем мобильного приложения. Подобный метод расстановки событий с точки зрения программного кода не является новым для разработчиков и предлагается существующими аналитическими платформами, однако интерпретация полученных результатов и последующие действия компании часто становятся серьезной проблемной задачей. Дальнейшее развитие такой методологии, основанной на аналитике, сегментации и обзорах пользователей, может разрешить задачу создания компаниями - разработчиками мобильных приложений собственной выверенной на практике маркетинговой стратегии.

Ключевые слова: конкурентоспособность, расстановка событий, маркетинговые исследования, сегментация, когортный анализ.

\title{
METHODS OF RESOLVING PROBLEMS CONNECTED WITH RAISING COMPETITIVENESS OF MOBILE APPLICATION
}

\author{
Irina P. Lebedeva \\ Plekhanov Russian University of Economics, \\ Moscow, Russia
}

Problems that should be resolved by marketer when a new mobile application is put on sale can be divided into two groups. The 1-st one is connected with customer attraction, the 2-nd one - with his/her retaining. On the one hand, resolving these problems is directly connected with advertizing campaign and marketing research, however the majority of companies - developers of mobile applications have no financial possibilities for marketing accompaniment of application entering market. The article studies research, which can be done by own forces. With adequate analysis they can lead to high-quality results and increase in the number of users. Apart from that the author proposes the method of analyzing event arrangement in application software section, which can help keep an eye on user's steps and interpret them to find key problems of the application. This method of event arrangement is not new for developers and it is offered by existing analytical platforms, however interpretation of obtained results and subsequent company steps can become a serious task. Further development of such methodology based on analytics, segmentation and reviews of users could resolve a problem of developing by companies-developers of mobile applications their own marketing strategy verified in practice.

Keywords: competitiveness, event arrangement, marketing research, segmentation, cohort analysis. 


\section{Введение}

$\Pi$ ри запуске любого мобильного приложения главная задача разработчика состоит в постоянном привлечении пользователей и, как следствие, в обеспечении его окупаемости. Однако постоянное привлечение пользователей за счет рекламы далеко не всегда является правильным маркетинговым ходом IT-компаний, специализирующихся на мобильном рынке. Проблема заключается в том, что компании выпускают на рынок большое количество приложений и вкладывание финансовых средств в несколько рекламных кампаний на постоянной основе при отсутствии достаточной прибыли не представляется возможным.

По данным компании Liftoff 1 , в 2019 г. произошло значительное снижение количества мобильных приложений по сравнению с предыдущим годом. В неигровых категориях новых приложений также заметен спад. Новых приложений в категории «Путешествия» стало на $40 \%$ меньше, социальных приложений - на 32,3\% меньше, финансовых - на 25,5\%, в категории «Покупки» - на 16,7\%. Это свидетельствует о том, что добиться внимания пользователей разработчикам становится все труднее.

По данным компании Omni Solutions², одним из основных факторов, сдерживающих рост рынка мобильных приложений в России, можно назвать высокую стоимость разработки - от 3 до 15 млн рублей, которую не все ретейлеры могут себе позволить. Кроме того, для успешного выхода на мобильный рынок нужна грамотная маркетинговая кампания, требующая дополнительных вложений. При этом речь идет именно о ретейлерах - устоявшихся компаниях, получающих стабильную прибыль от продаваемой продукции через Интернет или в магазинах.

\footnotetext{
1 URL: https://liftoff.io/blog/2019-mobile-app-trendsreport/(дата обращения: 08.02.2021).

2 URL: http://omni-solutions.ru/ (дата обращения: 08.02.2021)
}

\section{Очевидные проблемы и задачи продвижения мобильных приложений для компаний-разработчиков}

Исследуемый сектор мобильного рынка - мелкие и средние IT-компании и стартапы, создающие приложения, которые нацелены на привлечение клиентов за счет предложения услуги, потенциально востребованной у потребителя, и, как следствие, - дальнейшее развитие. Таким образом, разработчик данного сектора получит прибыль только в том случае, если выпущенное приложение будет признано пользователем. Соответственно, денежных средств для поддержания рекламной кампании у разработчиков приложений рассматриваемого сектора нет.

Отдельный сектор мобильного рынка составляют крупные устоявшиеся компании, которые занимаются выкупом прав на приложения у мелких и средних компаний и в дальнейшем используют рекламу как вложение денежных средств для продвижения приложений [9].

Таким образом, задачей разработчика становится привлечение пользователей без затрат на рекламу. Эта глобальная задача делится на две основные: улучшить мнение о приложении на рынке и удержать скачавшего приложение пользователя. Все остальные задачи носят опосредованный характер. Оба вопроса являются взаимосвязанными и зависимыми друг от друга.

\section{Мнение о приложении на рынке: количество скачиваний, качество отзывов, место в поиске}

Первое, на что обращают внимание пользователи, занимаясь поиском приложения по заданному функционалу, - это отзывы, которые оставили пользователи, уже скачавшие это приложение. Таким образом, задачей разработчика становится увеличение проставленной оценки и повышение качества отзывов. При наличии высоких оценок и хороших отзывов это становится бесплатной рекламой для приложения.

Вместе с тем стоит учитывать, что даже при наличии высоких оценок в случае не- 
большого количества пользователей приложение может оказаться в конце списка себе подобных в аналогичной категории и пользователь не сможет его заметить. По результатам проведенных исследований при выборе приложения пользователь обращает внимание на первые 5-10 предложений в списке. При этом количество приложений в одной и той же категории в десятки раз больше. Для примера, количество приложений, указывающих точное время в Google Market, - 251 шт.

Таким образом, здесь имеет место задача удержания скачавшего приложение пользователя, поскольку увеличение количества пользователей повышает место приложения в рейтинге магазинов мобильных приложений в одной категории. Кроме того, для рейтинга магазинов мобильных приложений имеют значение отзывы, оставляемые пользователями [2].

Для выяснения мнения пользователя разработчики пытаются проводить миниопросы и стимулировать комментарии о себе в самих приложениях. При этом основное внимание отводится не на интерфейс, а на общий уровень баллов по 5-балльной шкале и оставленные отзывы.

\section{Удержание пользователя - сложная задача, включающая в себя множество неизвестных}

Удержание или вовлечение пользователей - это показатель того, насколько люди, загрузившие приложение, на самом деле его используют. Но настоящее понимание взаимодействия с пользователем включает в себя измерение того, как люди его используют, как часто и как долго, до какой глубины, когда и почему. Кроме того, взаимодействие различается для разных категорий мобильных приложений. Можно сказать, что вовлечение пользователей является показателем, отражающим соответствие ключевым бизнес-целям разработчика приложений.

Для решения задачи удержания пользователя необходимо понять, что нравится или наоборот не нравится пользователю, каким образом увеличить длительность пользования приложением. Однако проводить маркетинговые исследования в данном случае представляется сложной задачей, так как количественные исследования требуют погружения в вопрос достаточного для получения репрезентативной выборки количества пользователей. Необходимо изучение ими приложения в течение некоторого времени, а реальные пользователи, скачавшие приложение, отвечать на вопросы не любят.

Самый простой способ получения информации - использование в приложении мобильных инструментов вовлечения (опросники, сообщения и баннеры), однако количество ответов сведется к числу, соответствующему отзывам, а качество при небольшом количестве пользователей будет оставаться под сомнением.

Возможно проведение фокус-групп, но в данном случае существует опасность сведения всех приложений к определенному однообразному виду: пользователь будет стремиться описать разработчику принцип действия или внешний вид своего любимого уже существующего приложения. Вместе с тем не факт, что, получив приложение, описанное им в фокус-группе, пользователь продолжит им пользоваться. Наиболее предпочтительны в данном случае смешанные приемы проведения маркетинговых исследований, сочетающие в себе как качественные, так и количественные методы, но для каждого приложения и каждого возникающего у разработчика вопроса они будут различны [5].

Одним из самых простых способов сбора информации для разработчика является просмотр и анализ отзывов пользователей, которые они оставляют в магазине приложений [3].

Еще один результативный метод получения информации - сегментация пользователей. Анализ поведения различных групп и их потребностей может значительно упростить разработчику мобильного приложения задачу выявления предпочтений пользователей и улучшить работу приложения [1]. 
По статистике компании Localytics ${ }^{1}$, $61 \%$ пользователей в среднем не будут пользоваться приложением больше месяца и всего $22 \%$ пользователей будут продолжать использовать его более трех месяцев. Один из видов сегментации направлен именно на анализ и решение задачи увеличения длительности пользования приложением. Когортный анализ позволяет делить пользователей на когорты (группы) по дате подключения и отслеживать, какое количество пользователей, подключившихся в определенный день, остается в приложении со временем. Этот вид сегментации наиболее наглядно отображает поведение пользователей в случае внесения изменений в приложение и демонстрирует их реакцию на произошедшие действия.

\section{Метод сбора аналитических данных путем расстановки событий}

Можно практическим путем определить информативный ряд показателей, которые демонстрируют привлекательность и эффективность работы приложения, а также его конкурентоспособность. Для этого используется вариант расстановки событий (маркеров) на определенных этапах приложения.

Все этапы приложения содержат набор задач для пользователя, каждая из которых характеризуется определенными действиями. Каждое событие является показателем выполнения (или невыполнения) какоголибо определенного действия пользователя.

Если подходить упрощенно, то можно сказать, что этап - это логически законченный эпизод приложения, который может содержать несколько страниц. Задача это набор действий пользователя, необходимых для прохождения данного этапа. Этап может состоять из одной или нескольких задач, а для выполнения задачи может требоваться от одного до нескольких действий.

1 URL: http://omni-solutions.ru/ (дата обращения: 08.02.2021).
Суммарно события, отображающие действия пользователя при выполнении определенной задачи на каждом этапе, дают представление о его поведении и являются показателем работы мобильного приложения на данном этапе.

Задачи находятся на любой странице приложения. Самая простая задача - переход на следующую страницу. Эта задача может состоять из нескольких действий (переход на заданную страницу, выход, клик на нужную кнопку, выход из приложения и т. д.). Суммируя произошедшие события, можно проследить поведение пользователя при выполнении данной задачи.

Допустим, необходимо, чтобы в приложении пользователь на странице, следующей после входа, вошел в пользовательское соглашение и одобрил его. Положительный результат - согласие, отрицательный выход со страницы пользовательского соглашения, что приравнивается к выходу из приложения. В данном случае этап - это соглашение, задача - одобрение соглашения, содержащее набор возможных действий: зайти на страницу соглашения, одобрить соглашение, подтвердить одобрение соглашения, вернуться на предыдущую страницу без одобрения соглашения. Для каждого действия устанавливается событие, которое отражает действие пользователя. Эта информация помогает в дальнейшем при попытках изменить возможности программы для повышения процента удержанных пользователей.

Существуют стандартные события, которые предусматриваются разработчиком заранее и устанавливаются на этапе разработки приложения, и события, которые указывают маркетологи в процессе работы приложения для определения тактики в удержании пользователя.

Как показано в табл. 1, общее количество событий для среднего по размерам приложения рассчитывается в миллионах на 100 тыс. пользователей. 
Т а блица 1

Количество событий для одного приложения по данным Google Analytics (наиболее популярные 8 позиций)*

\begin{tabular}{|l|c|c|}
\hline \multicolumn{1}{|c|}{ Название события } & Количество событий & Всего пользователей \\
\hline 1. Пользовательское соглашение & 26855878 & 82647 \\
\hline 2. Старт сессии & 2497685 & 81917 \\
\hline 3. Просмотр экрана & 1587136 & 81628 \\
\hline 4. Freestyle adv v3 & 1509854 & 8260 \\
\hline 5. Freestyle market bg & 981032 & 14925 \\
\hline 6. Freestyle market & 845433 & 52407 \\
\hline 7. Быстрый запуск стиля & 309435 & 44432 \\
\hline 8. Freestyle paramount & 169694 & 24204 \\
\hline Итого & 35243034 & 85390 \\
\hline
\end{tabular}

* Источник: URL: https://analytics.google.com/analytics/web (дата обращения: 17.01.2021).

Такие события, как одобрение пользовательского соглашения, просмотр предложенного экрана, выбор конкретного пункта из предложенного списка, маркетолог устанавливает с самого начала. Однако в процессе работы приложения выясняется, что пользователь не добирается до середины работы приложения. Проблем может быть несколько:

1. Проблемы в работе программной части приложения.

2. Скучный интерфейс - затянутый описательный процесс, замедленное время работы.

3. Непонятный интерфейс - пользователь не понимает, где нажимать кнопку, что делать дальше, есть отзыв приложения на произведенное им действие или нет.

В таких случаях первоочередным решением становится поиск того места в программе приложения, при постановке маркера на которое был бы получен ответ на вопрос, какого рода проблема стоит перед разработчиком.

\section{Пример размещения событий \\ и варианты их интерпретации}

Рассмотрим простой случай применения и использования событий. Например, можно предположить, что в приложении на странице $X$ существует кнопка $Y$, нажимая на которую пользователь выполняет цель посещения данного приложения (кнопка вызова такси по заданному маршруту, кнопка подтверждения покупки, кнопка выбора интерфейса и т. д.). При этом достижение цели включает две задачи: 1) выбрать кнопку, ведущую к достижению цели; 2) дождаться ответа приложения.

Существует проблема, что со страницы $X$ пользователь уходит, не дождавшись момента достижения своей цели. События $A 1$ и $A 2$ заранее установлены на действия перехода пользователя на страницу $X$ и со страницы $X$ соответственно. В таком случае можно поставить событие $B$ на действие нажатия кнопки, а событие $C$ - на отображение подтверждения действия.

В случае, если событие $A 2$ равно событию $B$, ошибка является программной (рисунок).

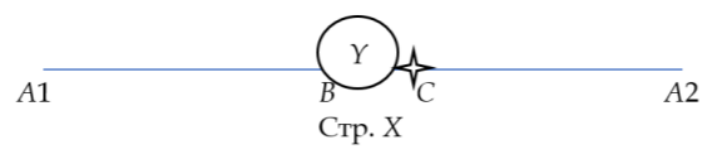

Рис. Отображение размещения событий на странице $X$ в схематичном виде

Случай, если событие A2 будет равно событию $C$, означает, что пользователь дожидается отзыва программы, но что-то мешает перейти ему на следующую страницу. Возможно, он считает, что программа окончила свое действие (непонятный интерфейс), возможно, время работы затянуто (скучный интерфейс). В первом случае необходимо вносить пояснения на страницу приложения, во втором случае соотнести временные затраты пользовате- 
ля на всю страницу и временные затраты пользователя между событиями С и $A 2$.

Данный случай описан как идеальный вариант сопоставления разных событий для отображения различных проблем, которые можно выявить подобным путем. Такие ситуации легко устраняются. При этом несложно выявить и другие ситуации, которые могут стать причиной покидания пользователем приложения.

На практике, если исключить программную ошибку, количество событий $A$, $B$ и $C$, скорее всего, не будут равны между собой. Это связано со многими факторами: различными психологическими свойствами пользователей, различным уровнем замотивированности достижения результата в данную минуту и посредством приложения, техническими качествами телефона и т. д. Например, реальное время, проходящее между событиями $B$ и $C$, составляет $T$ секунд. Исходя из практических заключений для интернет-приложения - это долгий срок. В результате пользователи разделятся на две группы (табл. 2).

\section{Деление пользователей на группы по принципу действия}

\begin{tabular}{|l|l|}
\hline \multicolumn{1}{|c|}{ Пользователи } & \multicolumn{1}{c|}{ Причины } \\
\hline $\begin{array}{l}\text { Группа 1. Дождется } \\
\text { отклика программы }\end{array}$ & $\begin{array}{l}\text { • Необходимость срочно получить положительный результат здесь и сейчас. } \\
\text { • Отсутствие спешки. } \\
\text { • Личностные качества: спокойный, сдержанный темперамент }\end{array}$ \\
\hline $\begin{array}{l}\text { Группа 2. Не дождется } \\
\text { отклика программы }\end{array}$ & $\begin{array}{l}\text { • Отсутствие необходимости срочно получить положительный результат. } \\
\text { • Уверенность в отсутствии смысла ожидания отклика программы после опре- } \\
\text { деленного промежутка времени (затянувшееся ожидание). } \\
\text { • Личностные качества: беспокойный, эмоциональный темперамент }\end{array}$ \\
\hline
\end{tabular}

В таком случае общее количество событий $B$ составит сумму свершившихся событий по группе 1 и группе 2 , а соотношение количества событий $B, C$ и $(B-C)$ может оказаться показательным для определения проблемы и ее дальнейшего решения:

$(B-C)>C$ - пользователи не готовы ждать отклика приложения длительное время ( $T$ секунд), время работы программы необходимо сокращать;

$(B-C)<C-$ пользователи готовы ждать отклика приложения $T$ секунд, можно рассматривать другие первостепенные задачи для улучшения работы приложения.

Уровень разницы между этими событиями во втором случае также можно рассматривать как степень готовности пользователей к ожиданию T секунд для получения ожидаемого результата в данном приложении, а следовательно, как лояльность пользователей к данному приложению.

Следуя этой же схеме, легко вычислить проблему с непонятным интерфейсом. Если поставить событие $A 1$ на вход на страницу $X$, то, скорее всего, при наличии не- понятного интерфейса количество событий $A 1$ будет больше числа событий $B$. Это связано с тем, что пользователь не понимает, как функционирует приложение. В таком случае следует заниматься упрощением приложения, увеличением наглядности его работы или размещением подсказок.

Таким образом, необходима разработка методики, которая могла бы служить подсказкой для разработчика и маркетолога, в каких местах следует расставлять события в определенных ситуациях. Правильно расставленные маркеры являются прекрасным способом выявления проблем, скрытых в приложении.

В 2020 г., несмотря на снижение количества мобильных приложений, количество их скачиваний в связи с мировой пандемией COVID-19 возросло. Исходя из этого у компаний-разработчиков появилась возможность увеличить число своих пользователей, а следовательно, увеличить и результативность упомянутых видов сбора информации. 


\section{Заключение}

Привлечение пользователей и их удержание, вовлечение в процесс пользования мобильным приложением - острый вопрос, который стоит перед всеми разработчиками мобильных приложений. Особенно это касается сектора рынка, не связанного с продажами товаров и услуг. В данном секторе невозможность проведения маркетинговых исследований и затраты на рекламу связаны с отсутствием финансовых средств в момент выпуска приложения на рынок. В этом случае средства для привлечения и удержания пользователей напрямую зависят от мнения и поведения уже имеющихся пользователей. От- слеживая их действия, можно выявить основные проблемы мобильного приложения и устранить их [10].

Предложенная схема интерпретации установленных событий позволяет аналитическим путем и без затрат на маркетинговые кампании выявить возникшие трудности. Дальнейшая разработка подобной методики, включая аналитику и отзывы пользователей, может оказаться альтернативой маркетинговым исследованиям, а при использовании сегментации - стать решением для разработчиков мобильных приложений при создании собственной маркетинговой стратегии.

\section{Список литературы}

1. Бородин А. И., Вейнберг Р. Р., ПисареВ Д. В., Литвишко О. В. Исследование остаточных артефактов Viber и Telegram в операционной системе Windows // Бизнес-информатика. 2019. - T. 13. - № 4. - С. 39-48.

2. Карпюк И. А., Куляшова Н. М. Сравнительный анализ мобильных приложений и инструментальных средств их разработки // Научно-методический электронный журнал «Концепт». - 2017. - Т. 31. - С. 826-830.

3. Макулин А. В., Рубенко А. С. «Серьезные игры»: мобильные приложения и «философия для всех» // Общество: философия, история, культура. - 2020. - № 6 (74). - С. 30-35.

4. Маркова Е. С., Кузнец,о М. М. Мобильные приложения как эффективный инструмент интернет-маркетинга // Актуальные проблемы и перспективы развития экономики: российский и зарубежный опыт. - 2020. - № 5 (30). - С. 30-34.

5. Мхитарян С. В. Аналитические компетенции маркетолога в условиях формирования цифровой экономики // Взгляд поколения XXI века на будущее цифровой экономики : сборник статей преподавателей IX Международной научно-практической конференции «Современная экономика: концепции и модели инновационного развития». М. : ФГБОУ ВО «РЭУ им. Г. В. Плеханова», 2018. - С. 227-232.

6. Мхитарян С. В., Данченок Л. А., Дейнекин Т. В. Методология маркетингового анализа в современных условиях : коллективная монография. - М. : МЭСИ, 2015.

7. Рольбина Е. С. Технология совмещения качественных и количественных методов исследования // Казанский экономический вестник. - 2017. - № 1 (27). - С. 104-109.

8. Сидорчук Р. Р., Коробков С. А., Мхитарян С. В. Использование интернет-ресурсов и мобильных приложений потребителями // Маркетинг и маркетинговые исследования. 2017. - № 1 (127). - C. 24-33.

9. Чернышева А. М., Долгова А. В. Организация маркетингового исследования в компании на современном этапе развития маркетинга // Наука и бизнес: пути развития. 2017. - № 4 (70). - C. 41-43.

10. Bentz B. Relevance Raises Response: How to Engage and Acquire with Mobile Marketing. - United States : SkillBites LLC, 2016. 


\section{References}

1. Borodin A. I., Veynberg R. R., Pisarev D. V., Litvishko O. V. Issledovanie ostatochnykh artefaktov Viber i Telegram v operatsionnoy sisteme Windows [Studying Residual Artifacts of Viber and Telegram in the Operational System Windows]. Biznes-informatika [BusinessInformatics], 2019, Vol. 13, No. 4, pp. 39-48. (In Russ.).

2. Karpyuk I. A., Kulyashova N. M. Sravnitelnyy analiz mobilnykh prilozheniy i instrumentalnykh sredstv ikh razrabotki [Comparative Analysis of Mobile Applications and Instrumental Means of their Development]. Academic Methodological E-Journal 'Concept', 2017, Vol. 31, pp. 826-830. (In Russ.).

3. Makulin A. V., Rubenko A. S. «Sereznye igry»: mobilnye prilozheniya i «filosofiya dlya vsekh» ['Serious Games': Mobile Applications and 'Philosophy for Everyone']. Obshchestvo: filosofiya, istoriya, kultura [Society: Philosophy, History, Culture], 2020, No. 6 (74), pp. 30-35. (In Russ.).

4. Markova E. S., Kuznetsov M. M. Mobilnye prilozheniya kak effektivnyy instrument internet-marketinga [Mobile Applications as Effective Tool of Internet-Marketing]. Aktualnye problemy $i$ perspektivy razvitiya ekonomiki: rossiyskiy $i$ zarubezhnyy opyt [Acute Problems and Prospects of Economy Development: Russian and Overseas Experience], 2020, No. 5 (30), pp. 30-34. (In Russ.).

5. Mkhitaryan S. V. Analiticheskie kompetentsii marketologa v usloviyakh formirovaniya tsifrovoy ekonomiki [Analytical Competences of Marketer in Conditions of Digital Economy Shaping]. View of Generation of the 21st Century on the Future of Digital Economy: collection of articles by lecturers of the 9th International Conference 'Today's Economics: Concepts and Models of Innovation Development'. Moscow, FGBOU VO «REU im. G. V. Plekhanovap», 2018, pp. 227232. (In Russ.).

6. Mkhitaryan S. V., Danchenok L. A., Deynekin T. V. Metodologiya marketingovogo analiza v sovremennykh usloviyakh, kollektivnaya monografiya [Methodology of Marketing Analysis in Present Day Conditions, collective monograph]. Moscow, MESI, 2015. (In Russ.).

7. Rolbina E. S. Tekhnologiya sovmeshcheniya kachestvennykh i kolichestvennykh metodov issledovaniya [Technology of Combining Qualitative and Quantitative Methods of Research]. Kazan Economic Bulletin, 2017, No. 1 (27), pp. 104-109. (In Russ.).

8. Sidorchuk R. R., Korobkov S. A., Mkhitaryan S. V. Ispolzovanie internet-resursov i mobilnykh prilozheniy potrebitelyami [The Use of Internet-Resources and Mobile Applications by Customers]. Marketing i marketingovye issledovaniya [Marketing and Marketing Research], 2017, No. 1 (127), pp. 24-33. (In Russ.).

9. Chernysheva A. M., Dolgova A. V. Organizatsiya marketingovogo issledovaniya v kompanii na sovremennom etape razvitiya marketinga [Organization of Marketing Research in Company on the Current Stage of Marketing Development]. Nauka i biznes: puti razvitiya [Science and Business: Ways of Development], 2017, No. 4 (70), pp. 41-43. (In Russ.).

10. Bentz B. Relevance Raises Response: How to Engage and Acquire with Mobile Marketing. United States, SkillBites LLC, 2016.

\section{Сведения об авторе}

Ирина Павловна Лебедева

аспирантка кафедры маркетинга

РЭУ им. Г. В. Плеханова.

Адрес: ФГБОУ ВО «Российский экономический университет имени Г. В. Плеханова», 117997,

Москва, Стремянный пер., д. 36.

E-mail: Lebedeva.IP@rea.ru

\section{Information about the author}

Irina P. Lebedeva

Post-Graduate Student of the Department

for Marketing of the PRUE.

Address: Plekhanov Russian University

of Economics, 36 Stremyanny Lane,

Moscow, 117997, Russian Federation.

E-mail: Lebedeva.IP@rea.ru 